\title{
Machine Recognition of Sounds in Mixtures
}

\section{Outline}

1 Computational Auditory Scene Analysis

(2) Speech Recognition as Source Formation

(3) Sound Fragment Decoding

(4) Results \& Conclusions

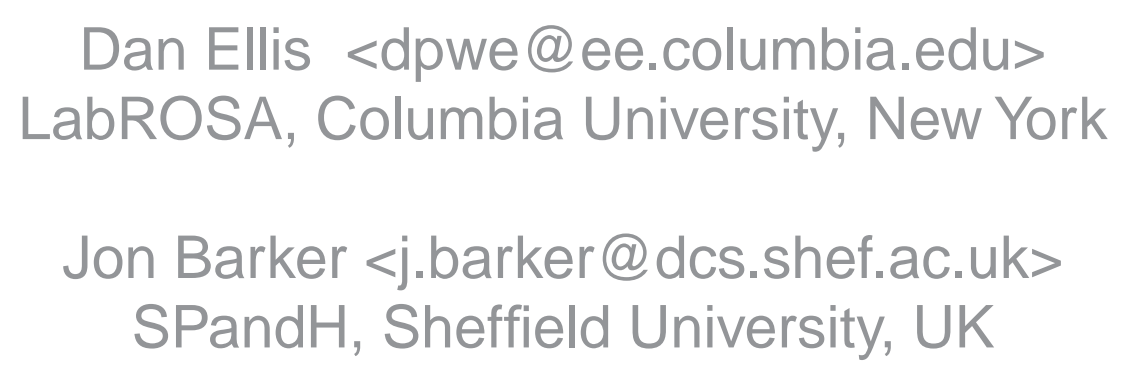




\section{(1) Computational Auditory Scene Analysis (CASA)}

- Human sound organization:

Auditory Scene Analysis

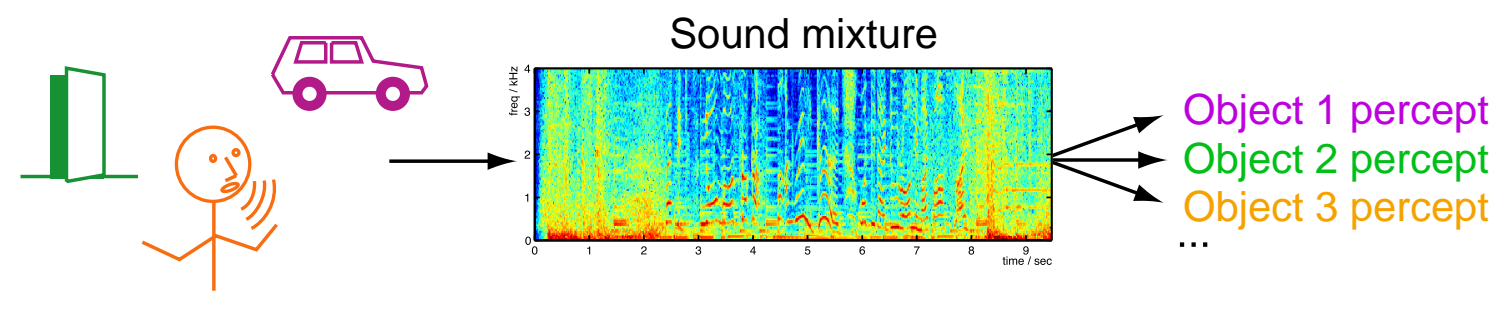

- composite sound signal $\rightarrow$ separate percepts

- based on ecological constraints

- acoustic cues $\rightarrow$ perceptual grouping

- Computational ASA:

Doing the same thing by computer

...? 


\section{What is the goal of CASA?}

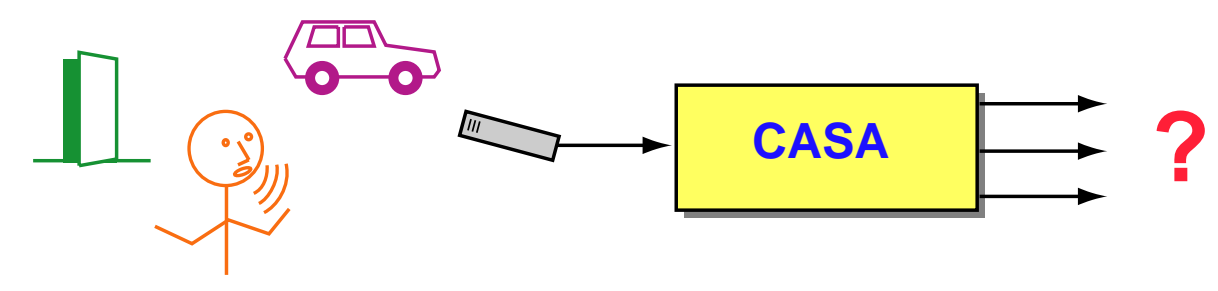

- Separate signals?

- output is unmixed waveforms

- underconstrained, very hard ...

- too hard? not required?

- Source classification?

- output is set of event-names

- listeners do more than this...

- Something in-between? Identify independent sources + characteristics

- standard task, results? 


\section{Segregation vs. Inference}

- Source separation requires attribute separation

- sources are characterized by attributes (pitch, loudness, timbre + finer details)

- need to identify \& gather different attributes for different sources ...

- Need representation that segregates attributes

- spectral decomposition

- periodicity decomposition

- Sometimes values can't be separated

- e.g. unvoiced speech

- maybe infer factors from probabilistic model?

$$
p(O, x, y) \rightarrow p(x, y \mid O)
$$

- or: just skip those values, infer from higher-level context 


\section{Outline}

(1) Computational Auditory Scene Analysis

(2) Speech Recognition as Source Formation

- Standard speech recognition

- Handling mixtures

(3) Sound Fragment Decoding

(4) Results \& Conclusions 

as Source Formation

- Automatic Speech Recognition (ASR): the most advanced sound analysis

- ASR extracts abstract information from sound

- (i.e. words)

- even in mixtures (noisy backgrounds) .. a bit

- ASR is not signal extraction: only certain signal information is recovered

- .. just the bits we care about

- Not CASA preprocessing for ASR: Instead, approach ASR as an example of CASA

- words $=$ description of source properties

- uses strong prior constraints: signal models

- but: must handle mixtures! 


\section{How ASR Represents Speech}

- Markov model structure: states + transitions
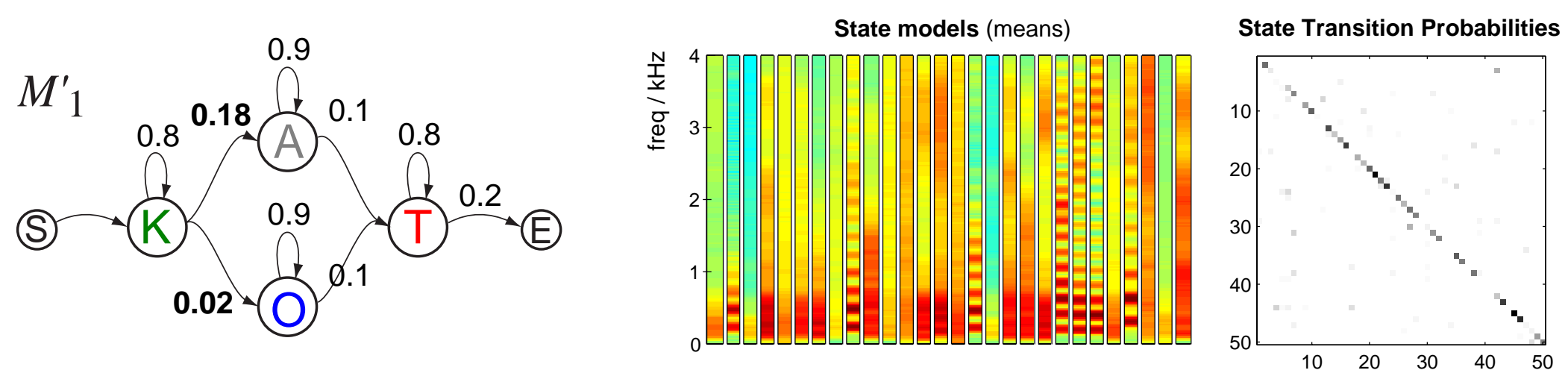

- Generative model

- but not a good speech generator!

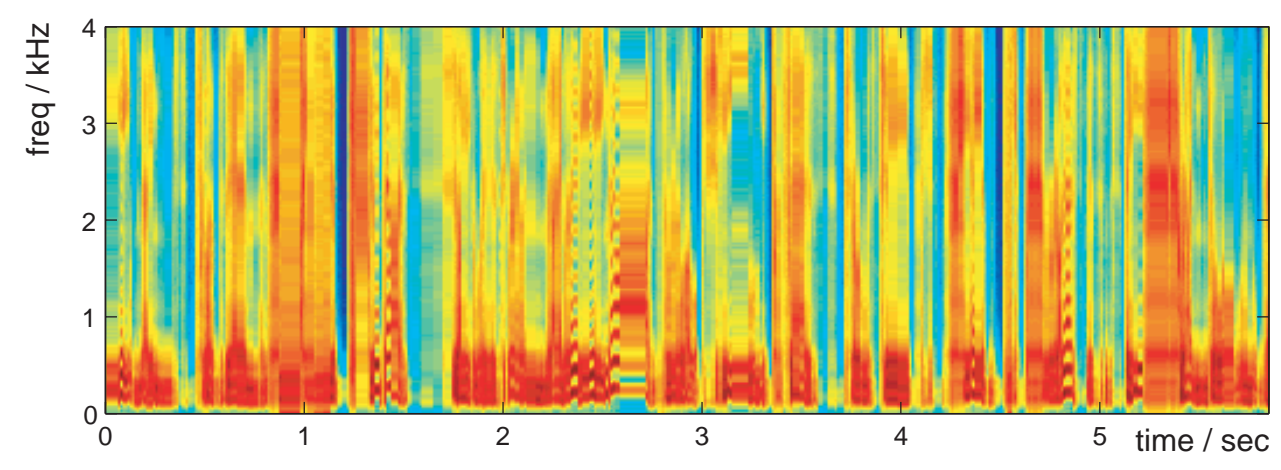

- only meant for inference of $p(X \mid M)$ 


\section{Sequence Recognition}

- Statistical Pattern Recognition:

$M^{*}=\underset{M}{\operatorname{argmax}} P(M \mid X)=\underset{M}{\operatorname{argmax}} \frac{P(X \mid M) \cdot P(M)}{P(X)}$

- Markov assumption decomposes into frames:

$$
P(X \mid M)=\prod_{n} p\left(x_{n} \mid m_{n}\right) p\left(m_{n} \mid m_{n-1}\right)
$$

- Solve by searching over all possible state sequences $\left\{m_{n}\right\} .$. but with efficient pruning:

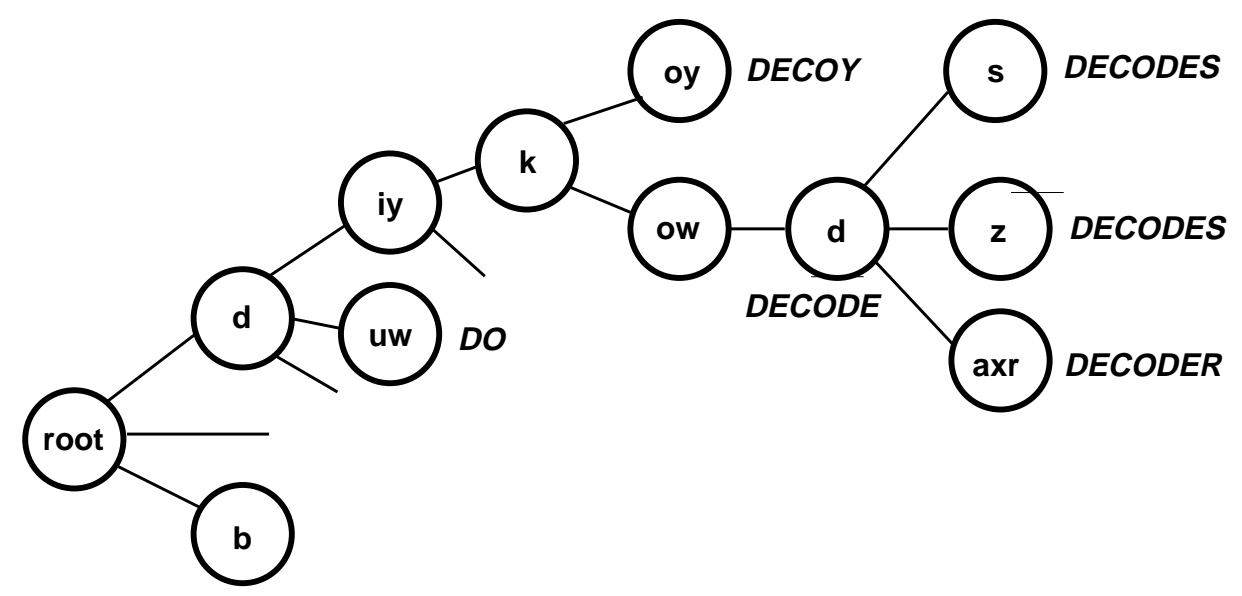




\section{Approaches to sound mixture recognition}

- Separate signals, then recognize

- e.g. (traditional) CASA, ICA

- nice, if you can do it

- Recognize combined signal

- 'multicondition training'

- combinatorics.

- Recognize with parallel models

- full joint-state space?

- divide signal into fragments, then use missing-data recognition 


\section{Outline}

(1) Computational Auditory Scene Analysis

(2) Speech Recognition as Source Formation

(3) Sound Fragment Decoding

- Missing Data Recognition

- Considering alternate segmentations

(4) Results \& Conclusions 


\section{Sound Fragment Decoding}

- Signal separation is too hard! Instead:

- segregate features into partially-observed sources

- then classify

- Made possible by missing data recognition

- integrate over uncertainty in observations for true posterior distribution

- Goal:

Relate clean speech models $P(X \mid M)$ to speech-plus-noise mixture observations

- .. and make it tractable 


\section{Missing Data Recognition}

- Speech models $p(\mathbf{x} \mid m)$ are multidimensional...

- i.e. means, variances for every freq. channel

- need values for all dimensions to get $p(\bullet)$

- But: can evaluate over a subset of dimensions $x_{k}$

$$
p\left(\mathbf{x}_{k} \mid m\right)=\int p\left(\mathbf{x}_{k}, \mathbf{x}_{u} \mid m\right) d \mathbf{x}_{u}
$$

- Hence, missing data recognition:
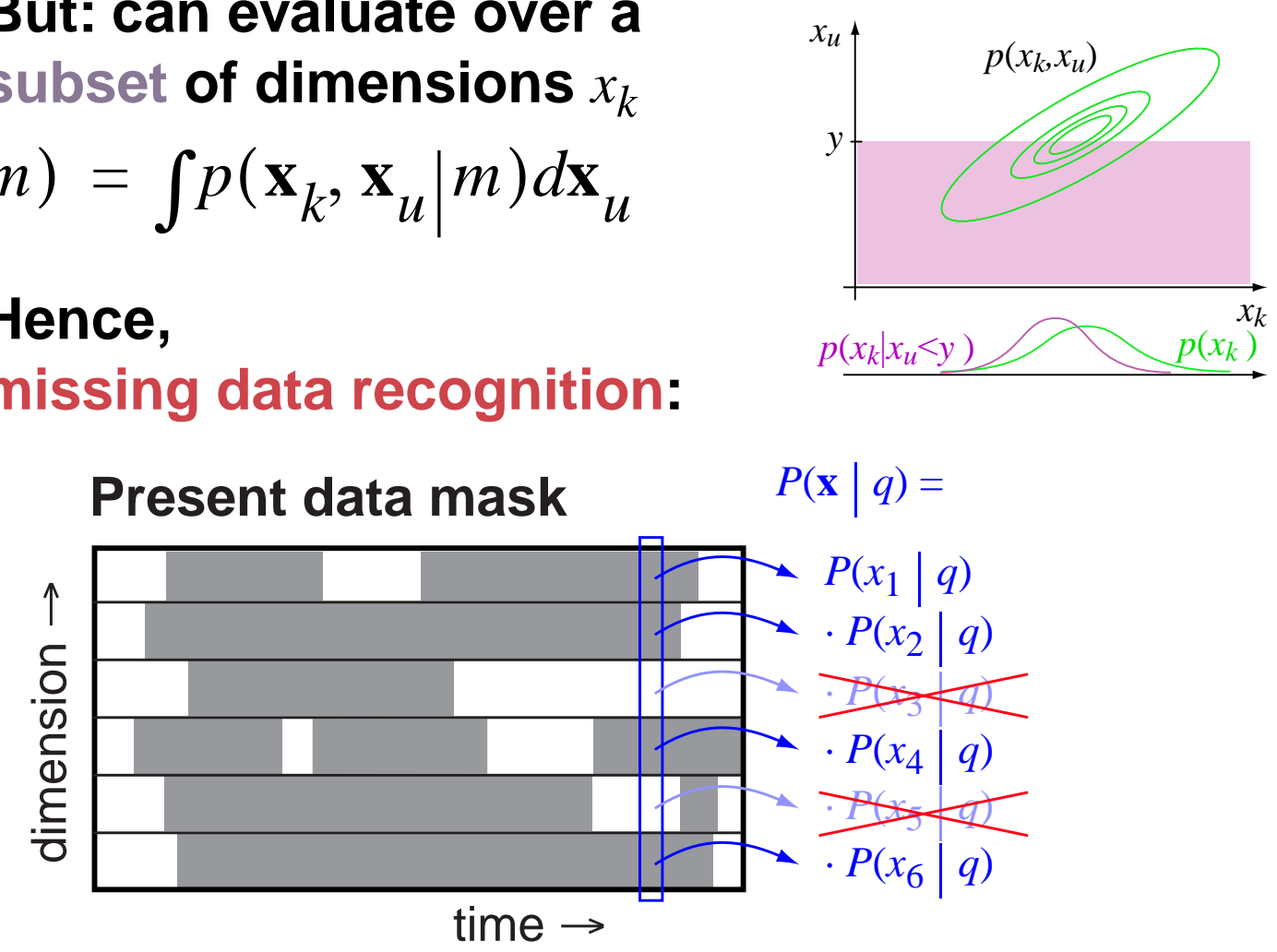

- hard part is finding the mask (segregation)

-




\section{Missing Data Results}

- Estimate static background noise level $N(f)$

- Cells with energy close to background are considered "missing"
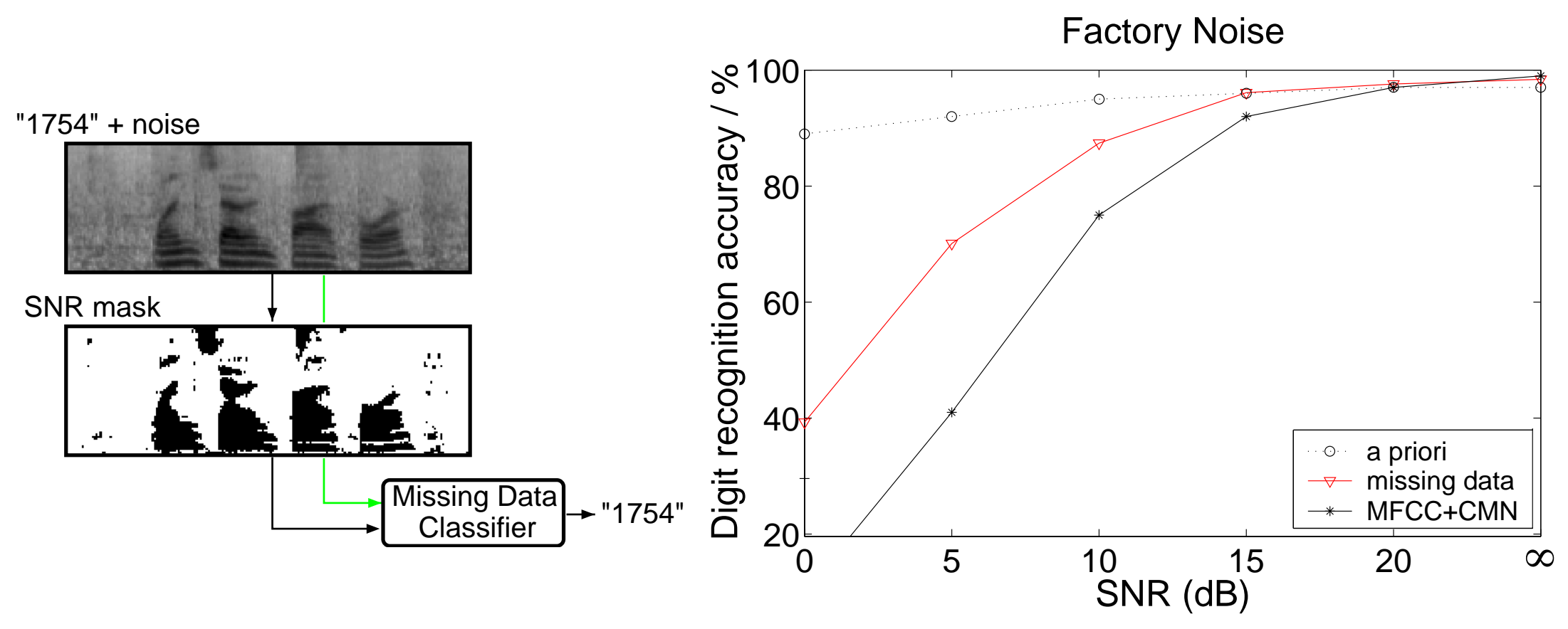

- must use spectral features!

- But: nonstationary noise $\rightarrow$ spurious mask bits

- can we try removing parts of mask? 


\section{Comparing different segregations}

- Standard classification chooses between models $M$ to match source features $X$

$$
M^{*}=\underset{M}{\operatorname{argmax}} P(M \mid X)=\underset{M}{\operatorname{argmax}} P(X \mid M) \cdot \frac{P(M)}{P(X)}
$$

- Mixtures: observed features $Y$, segregation $S$, all related by $P(X \mid Y, S)$

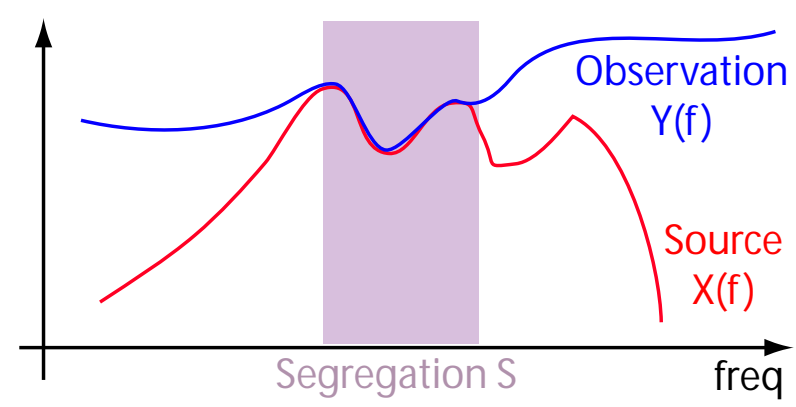

- Joint classification of model and segregation:

$$
P(M, S \mid Y)=P(M) \int P(X \mid M) \cdot \frac{P(X \mid Y, S)}{P(X)} d X \cdot P(S \mid Y)
$$

- $P(X)$ no longer constant 


\section{Calculating fragment matches}

$$
P(M, S \mid Y)=P(M) \int P(X \mid M) \cdot \frac{P(X \mid Y, S)}{P(X)} d X \cdot P(S \mid Y)
$$

- $\quad P(X \mid M)$ - the clean-signal feature model

- $\quad P(X \mid Y, S) / P(X)$ - is $X$ 'visible' given segregation?

- Integration collapses some bands...

- $P(S \mid Y)$ - segregation inferred from observation

- just assume uniform, find $S$ for most likely $M$

- or: use extra information in $Y$ to distinguish $S$ 's...

- Result:

- probabilistically-correct relation between clean-source models $P(X \mid M)$ and inferred, recognized source + segregation $P(M, S \mid Y)$ 


\section{Using CASA features}

- $\quad P(S \mid Y)$ links acoustic information to segregation

- is this segregation worth considering?

- how likely is it?

- Opportunity for CASA-style information to contribute

- periodicity/harmonicity: these different frequency bands belong together

- onset/continuity: this time-frequency region must be whole

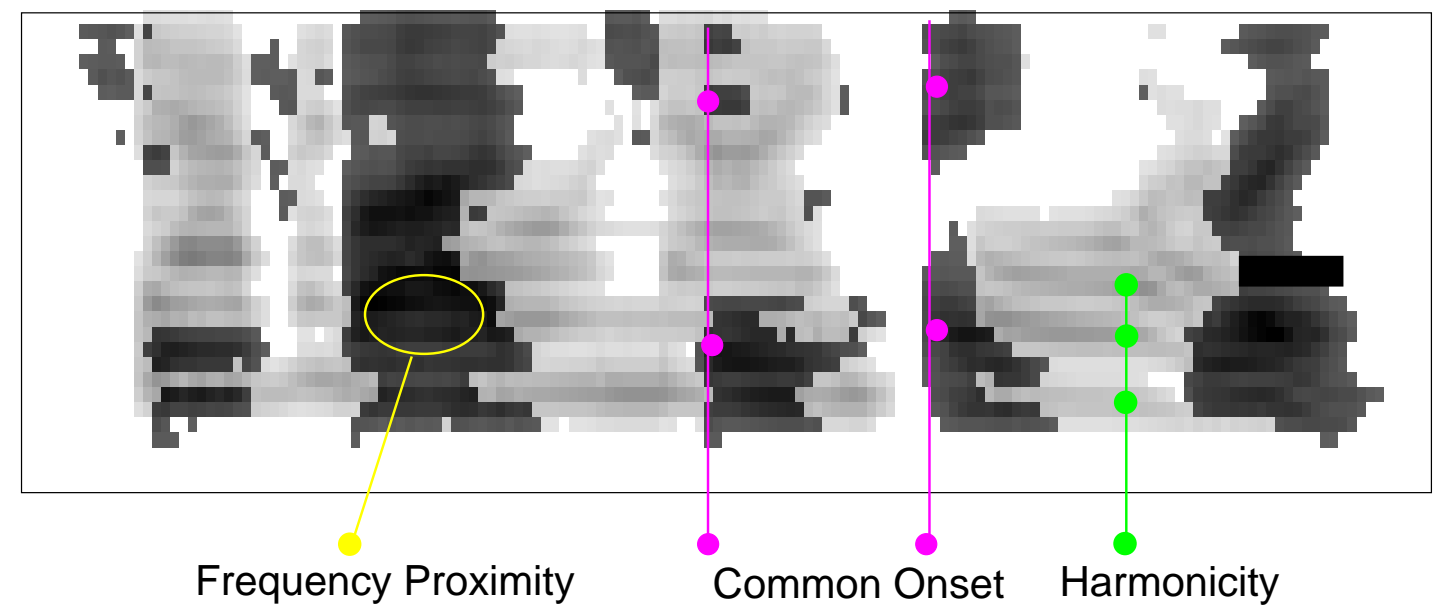




\section{Fragment decoding}

- Limiting $S$ to whole fragments makes hypothesis search tractable:

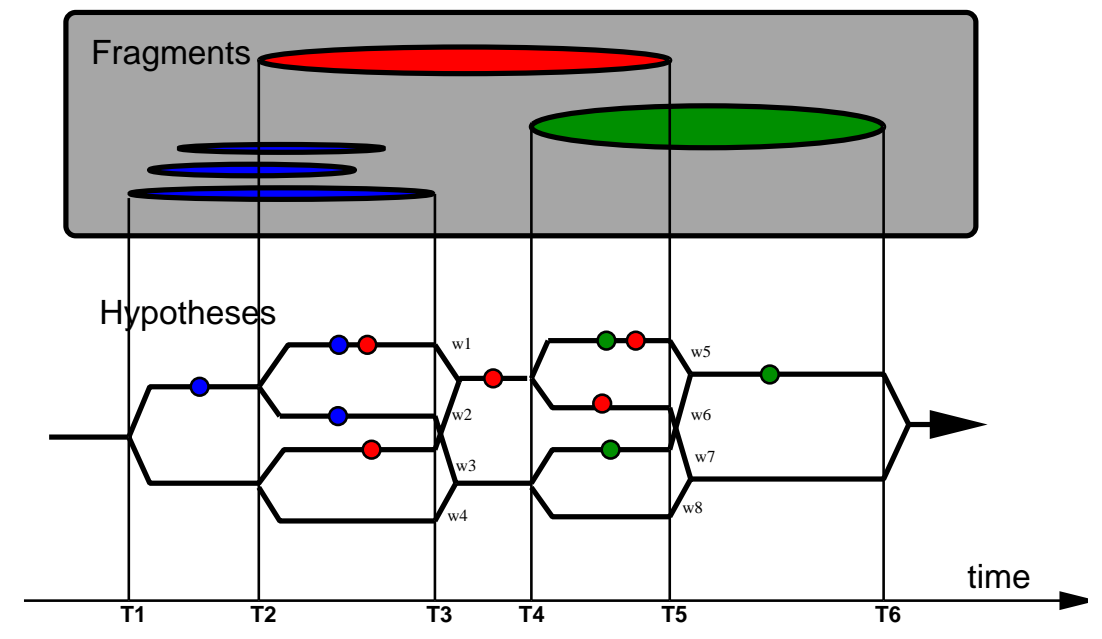

- choice of fragments reflects $P(S \mid Y) \cdot P(X \mid M)$ i.e. best combination of segregation and match to speech models

- Merging hypotheses limits space demands

- .. but erases specific history 


\section{Multi-Source Decoding}

- Match multiple models at once?

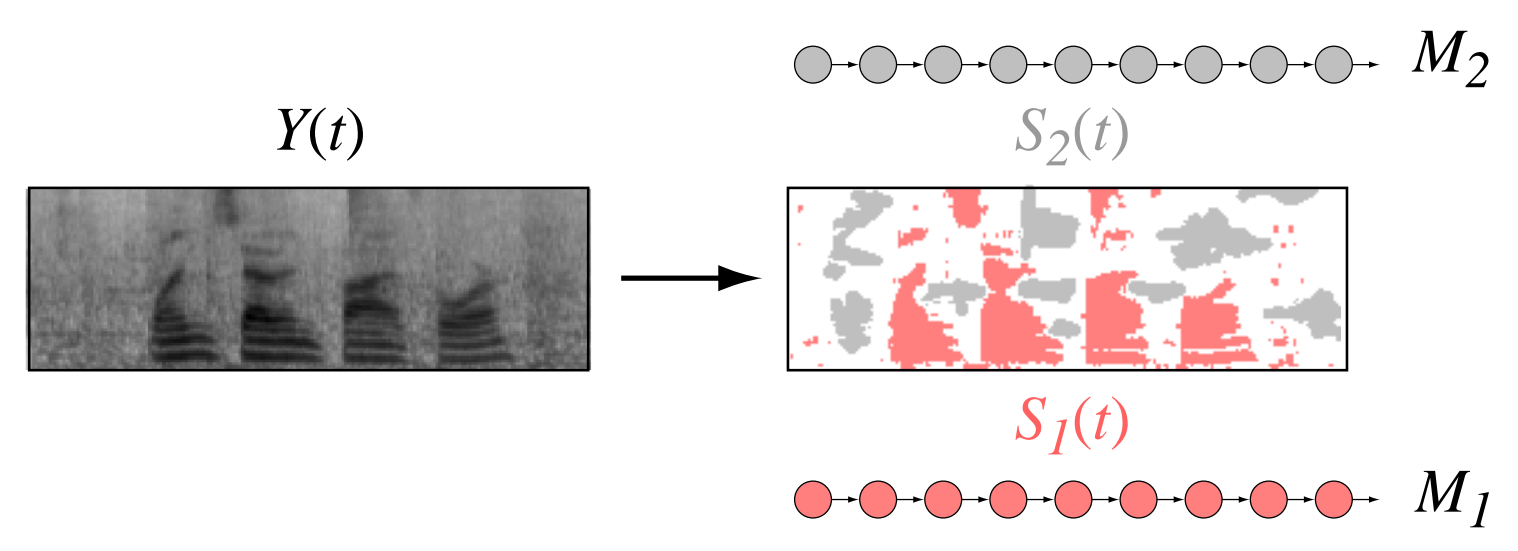

- disjoint subsets of cells for each source

- each model match $P\left(M_{x} \mid S_{x}, Y\right)$ is independent

- masks are mutually dependent: $P\left(S_{1}, S_{2} \mid Y\right)$ 


\section{Outline}

(1) Computational Auditory Scene Analysis

(2) Speech Recognition as Source Formation

(3) Sound Fragment Decoding

(4) Results \& Conclusions

- Speech recognition

- Alarm detection 


\section{(4) Speech fragment decoder results}

- Simple $P(S \mid Y)$ model forces contiguous regions to stay together

- big efficiency gain when searching $S$ space
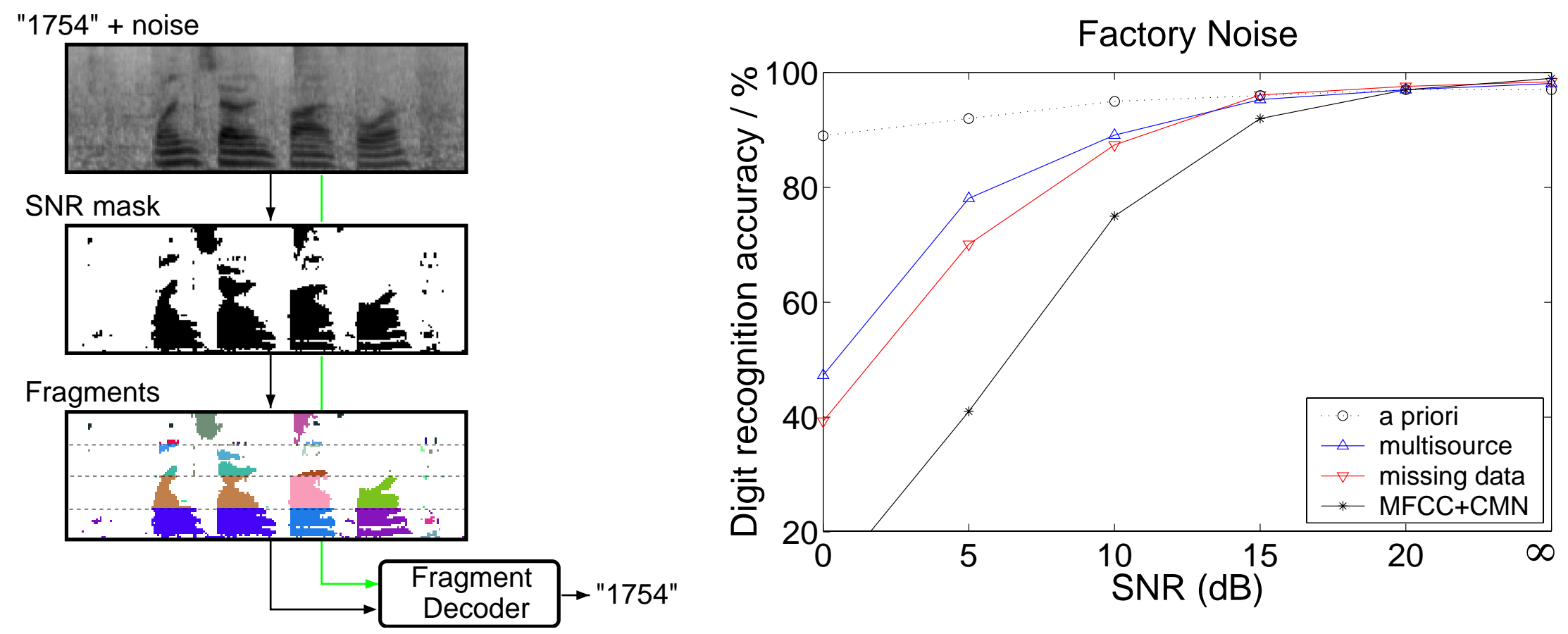

- Clean-models-based recognition rivals trained-in-noise recognition 


\section{Alarm sound detection}

- Alarm sounds have particular structure

- people 'know them when they hear them'

- clear even at low SNRs

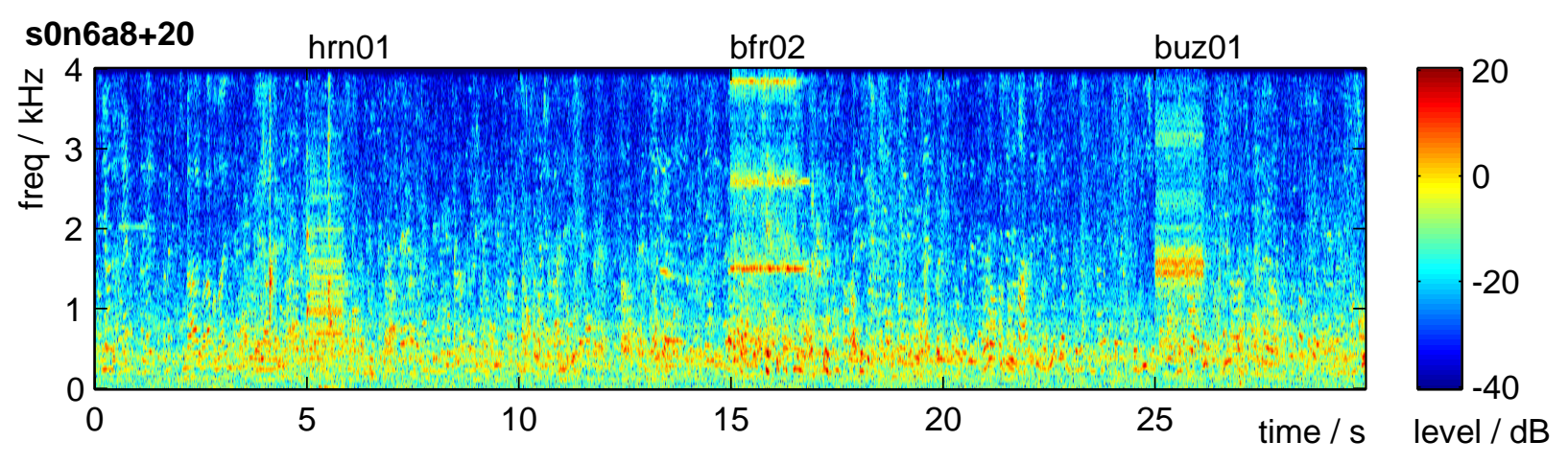

- Why investigate alarm sounds?

- they're supposed to be easy

- potential applications...

- Contrast two systems:

- standard, global features, $P(X \mid M)$

- sinusoidal model, fragments, $P(M, S \mid Y)$ 


\section{Alarms: Results}
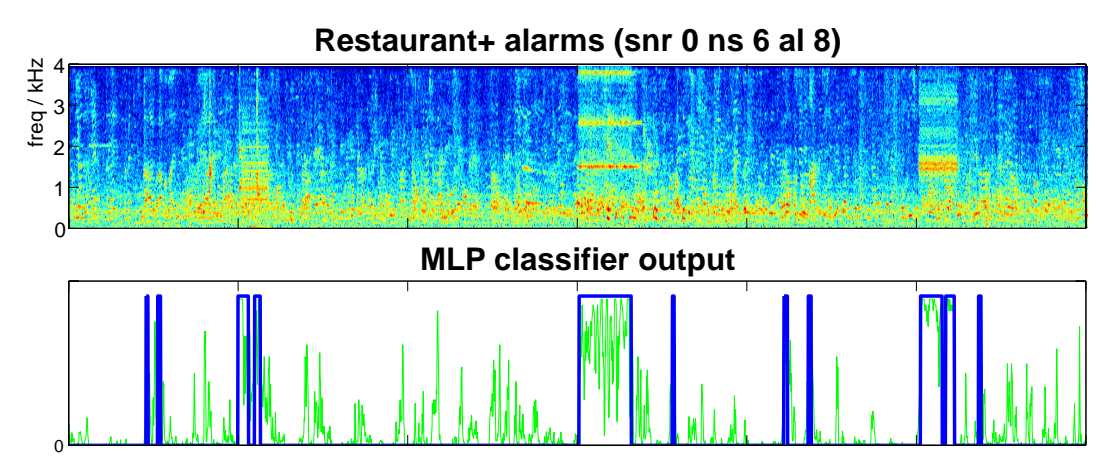

Sound object classifier output

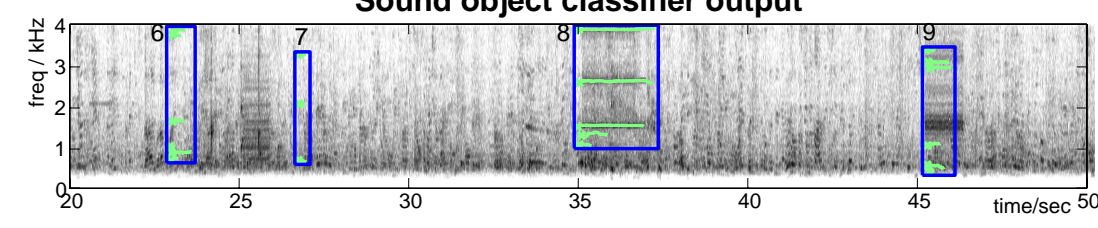

- Both systems commit many insertions at $0 \mathrm{~dB}$ SNR, but in different circumstances:

\begin{tabular}{|c|c|c|c|c|c|c|}
\hline \multirow{2}{*}{ Noise } & \multicolumn{3}{|c|}{ Neural net system } & \multicolumn{3}{|c|}{ Sinusoid model system } \\
\hline & Del & Ins & Tot & Del & Ins & Tot \\
\hline $1(\mathrm{amb})$ & $7 / 25$ & 2 & $36 \%$ & $14 / 25$ & 1 & $60 \%$ \\
\hline 2 (bab) & $5 / 25$ & 63 & $272 \%$ & $15 / 25$ & 2 & $68 \%$ \\
\hline 3 (spe) & $2 / 25$ & 68 & $280 \%$ & $12 / 25$ & 9 & $84 \%$ \\
\hline 4 (mus) & $8 / 25$ & 37 & $180 \%$ & $9 / 25$ & 135 & $576 \%$ \\
\hline Overall & $22 / 100$ & 170 & $192 \%$ & $50 / 100$ & 147 & $197 \%$ \\
\hline
\end{tabular}




\section{Summary \& Conclusions}

- Scene Analysis

- necessary for useful hearing

- Recognition

- a model domain for scene analysis

- Fragment decoding

- recognition with partial observations

- combines segmentation \& model fitting

- Future work

- models of sources other than speech

- simultaneous 'perception' of multiple sources 